\title{
Total Laparoscopic Hysterectomy for Adenomyosis in a Patient Receiving Peritoneal Dialysis: A Case Report
}

\author{
Shoko Ueda, Tomohito Tanaka*, Shunsuke Miyamoto, Yoshito Terai and Masahide Ohmichi \\ Department of Obstetrics and Gynecology, Osaka Medical College, Takatsuki, Japan \\ ${ }^{*}$ Correspondence author: N Tomohito Tanaka, M.D., Ph.D., 2-7, Daigaku-machi, Takatsuki, Osaka 569-8686, Japan; Tel: +81726-831221; \\ E-mail: gyn123@osaka-med.ac.jp
}

Received: October 17, 2018; Accepted: October 26, 2018; Published: November 10, 2018;

\begin{abstract}
Peritoneal dialysis is a common supportive therapy for chronic renal failure, whereas laparoscopic surgery has rarely been performed. We herein report laparoscopic hysterectomy in a patient receiving peritoneal dialysis. A 44-year-old patient receiving peritoneal dialysis underwent laparoscopic hysterectomy due to a large amount of vaginal bleeding caused by adenomyosis. At surgery, the peritoneum turned whitish with inflammation. The small intestine was adhered to the peritoneum, omentum or both; however, no serious adhesion was found in the pelvic cavity. After hysterectomy, the peritoneal defect was completely repaired out of consideration of the need for peritoneal dialysis after surgery. There were no complications during or after surgery. No peritoneal leakage was observed. Laparoscopic hysterectomy was suggested to be safe and feasible in patients who are receiving peritoneal dialysis.
\end{abstract}

Keywords: peritoneal dialysis, hysterectomy, laparoscopy, total laparoscopic hysterectomy, adenomyosis

\section{Introduction}

Peritoneal Dialysis (PD) is an established management for patients with stage 5 chronic kidney disease [1]. Although laparotomy has been considered the standard treatment for PD patient candidates for intra-abdominal surgery to reduce the risk of complications, a high incidence of perioperative complications, including dialysate fluid leakage, wound dehiscence, incisional hernia, peritonitis and hemoperitoneum, has been reported [2,3]. A recent study showed that laparoscopic surgery was well accepted as being a conservative procedure associated with a less-invasive approach, lower peritoneal membrane stress and better preservation of the peritoneum integrity than laparotomic surgery [4-6]. However, few reports have been published concerning laparoscopic hysterectomy for patients with PD.

We herein report a case of total laparoscopic hysterectomy for adenomyosis in a patient who was receiving PD.

\section{Case Presentation}

A 44-year-old nulliparous woman had a large amount of vaginal bleeding caused by adenomyosis. The patient had completely lost her renal function due to chronic renal failure and had been receiving PD for 7 years. While receiving PD, the patient had an irregular menstrual cycle and had not menstruated for the past six months. Transvaginal ultrasound and magnetic resonance imaging revealed that the patient had an enlarged uterus (the size of that at 12 weeks' gestation) that was suspected of being adenomyosis. An endometrial biopsy showed complex endometrial hyperplasia without atypia. After hospitalization, the patient received hemodialysis because she needed a blood transfusion and hydration. Laparoscopic hysterectomy was scheduled because the patient had a continuous large amount of vaginal bleeding despite hormone therapy.

Total laparoscopic hysterectomy with bilateral salpingooophorectomy was performed with pneumoperitoneum under general anesthesia. A 12-mm port was placed through the transumbilical incision for the operative laparoscope via the open method. Three ancillary 5-mm ports were positioned; two in each lower quadrant and one in the suprapubic area. The small intestine were found to be adhered to the peritoneum, omentum or both (Figure 1A); however, no serious adhesion was found in the pelvic cavity (Figure 1B). A catheter for PD had been placed through the left abdominal wall to the vesico-uterine pouch (Figure 1C). No adhesion was found around the PD catheter. Most of the peritoneum turned whitish. The peritoneum was dissected near the uterus in order to repair the peritoneum defect. At hysterectomy, a 5-cm transverse incision was made on the suprapubic area to remove the uterus. After hysterectomy, the opened peritoneum and retroperitoneum were completely sutured (Figure 1D). The operative duration was $183 \mathrm{~min}$ with an estimated blood loss of only a few milliliters. There were no perioperative complications, including dialysate fluid leakage, wound dehiscence, incisional hernia, peritonitis and hemoperitoneum.

PD was restarted on postoperative day 16. Pathologically, the uterus was diagnosed with adenomyosis. Six months have passed since the treatment, and PD has been performed as before surgery without issue. 


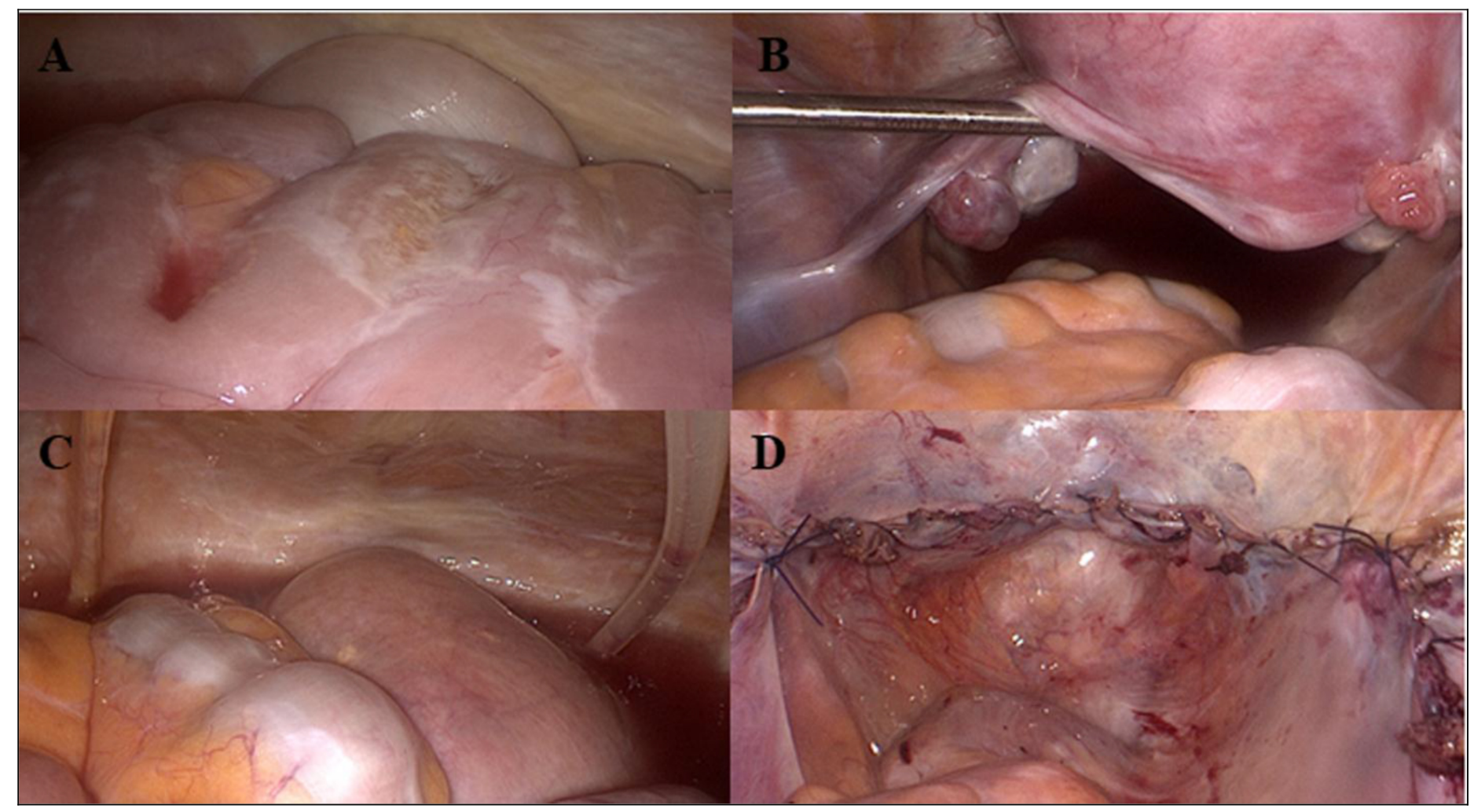

Figure 1. (A) At surgery, the small intestine was found to be adhered to the peritoneum, omentum or both.

(B) No serious adhesion was found in the pelvic cavity. The uterus was enlarged to the size of that at 12 weeks of gestation. (C) The catheter for PD had been placed through the left abdominal wall to the vesico-uterine pouch. (D) After hysterectomy, the opened peritoneum and retroperitoneum were completely sutured.

\section{Discussion}

In the current case, we performed laparoscopic hysterectomy for adenomyosis in a patient receiving PD. No perioperative complications were found because the peritoneal defect was completely repaired. PD was successfully restarted 16 days after surgery.

Generally, surgery in the peritoneal cavity for patients receiving PD is associated with complications, including leakage of dialysis fluid, infection and peritonitis. Some patients may have a decreased peritoneal clearance due to a postoperative peritoneal defect and leakage [7]. Laparoscopic surgery is well accepted as being a conservative procedure associated with a less-invasive approach, lower peritoneal membrane stress and better preservation of the peritoneum integrity than laparotomic surgery $[5,6]$. For these reasons, laparoscopic surgery has been a standard method for cholecystectomy, appendectomy and nephrectomy in patients with PD [7]. However, there are few reports concerning laparoscopic hysterectomy in patients receiving PD.

Kakuda et al. performed total laparoscopic hysterectomy for endometrial cancer in a renal transplant patient receiving PD. While they successfully performed the procedure, $\mathrm{PD}$ could not be restarted due to dialysate fluid leakage [8]. Lew et al. reported a case of roboticassisted total laparoscopic hysterectomy for endometrial cancer in a PD patient, and PD was able to be restarted three days after surgery, although the patient suffered from perioperative complications, including opiate-associated constipation and peritonitis [9].
In the current case, the peritoneum turned whitish with inflammation, and the small intestine was found to adhere to the peritoneum, omentum or both at surgery; however, no perioperative complications were noted after surgery, and PD was able to be restarted 16 days after surgery.

In conclusion, we performed laparoscopic hysterectomy for adenomyosis in a patient receiving PD without unexpected complications. The lack of perioperative complications thanks to the complete repair of the peritoneal defect enabled the patient to restart PD without issue.

\section{Acknowledgment}

The authors report no conflicts of interest. The authors alone are responsible for the content and writing of the paper. This case report was approved by a constituted ethics committee of our hospital, and it conforms to the provisions of the 1995 Declaration of Helsinki (as revised in Brazil 2013). Written informed consent was obtained from the patient, and patient anonymity was preserved.

\section{References}

1. Lukowsky LR, Mehrotra R, Kheifets L, Arah OA, Nissenson AR, et al. (2013) Comparing mortality of peritoneal and hemodialysis patients in the first 2 years of dialysis therapy: a marginal structural model analysis. Clinical journal of the American Society of Nephrology CJASN 8: 619-628.

2. Moffat FL, Deitel M, Thompson DA (1982) Abdominal surgery in patients undergoing long-term peritoneal dialysis. Surgery 92: 598-604. 
3. Rais-Bahrami S, Romero FR, Lima GC, Kohanim S, Kavoussi LR (2006) Reinstatement of continuous ambulatory peritoneal dialysis after transperitoneal laparoscopic nephrectomy. Urology 68: 715-717. [crossref]

4. Ha JF, Chandraratna H (2009) Laparoscopic cholecystectomy in chronic ambulatory peritoneal dialysis. The Ochsner journal 9: 17-19.

5. Kleinpeter MA, Krane NK (2006) Perioperative management of peritoneal dialysis patients: review of abdominal surgery. Advances in peritoneal dialysis Confe on Peritoneal Dialysis 22: 119-123.

6. Keshvari A, Fazeli MS, Meysamie A, Seifi S, Taromloo MK (2010) The effects of previous abdominal operations and intraperitoneal adhesions on the outcome of peritoneal dialysis catheters. Peritoneal dialysis international: journal of the International Society for Peritoneal Dialysis 30: 41-45.

7. Mari G, Scanziani R, Auricchio S, Crippa J, Maggioni D (2017) Laparoscopic Surgery in Patients on Peritoneal Dialysis: A Review of the Literature. Surgical innovation 24: 397-401.

8. Kakuda M, Kobayashi E, Tanaka Y, Ueda Y, Yoshino K, et al. (2017) Total laparoscopic hysterectomy for endometrial cancer in a renal transplantation patient receiving peritoneal dialysis: Case report and literature review. The journal of obstetrics and gynaecology research 43: 1232-1237.

9. Lew SQ, Chernofsky MR (2016) Uninterrupted Peritoneal Dialysis after RoboticAssisted Total Laparoscopic Hysterectomy. Peritoneal dialysis international: journal of the International Society for Peritoneal Dialysis 36: 349-350.

\section{Citation:}

Shoko Ueda, Tomohito Tanaka, Shunsuke Miyamoto, Yoshito Terai and Masahide Ohmichi (2018) Total Laparoscopic Hysterectomy for Adenomyosis in a Patient Receiving Peritoneal Dialysis: A Case Report. Integr Gyn Obstet J Volume 1(2): 1-3 